\title{
Symmetry database of International Tables online
}

\author{
E. Kroumova ${ }^{1}$, G. de la Flor ${ }^{2}$, N. J. Ashcroft ${ }^{3}$ and M. I. Aroyo ${ }^{4}$
}

\begin{abstract}
${ }^{1}$ eFaber Soluciones Inteligentes SL., Bilbao (Spain), ${ }^{2}$ Institute of Applied Geosciences, Karlsruhe Institute of Technology, Karlsruhe (Germany), ${ }^{3}$ Editorial Office, International Union of Crystallography, Chester (England), ${ }^{4}$ Departamento de Física, Universidad del País Vasco UPV/EHU, Bilbao (Spain)
\end{abstract}

\author{
mois.aroyo@ehu.es
}

The Symmetry Database (https://symmdb.iucr.org/) forms part of the online edition of International Tables for Crystallography and gives access to databases of crystallographic point and space groups. These online databases expand and complement the symmetry information provided in the print editions of International Tables for Crystallography Volume A, Space-Group Symmetry [1] or Volume A1, Symmetry Relations between Space Groups [2]. The information in the database can either be retrieved directly or generated 'on-the fly' using a range of programs. Help pages briefly explain the crystallographic data and the functionality of the programs. The data and programs that are currently available in the Symmetry Database are arranged into three sections:

(i) Space-group symmetry: The data in Volume A are extended to include the generators, general positions and Wyckoff positions of all 230 space groups, including the 530 settings for the monoclinic and orthorhombic space groups listed in Volume A. If data are not available for a particular setting directly, an arbitrary basis transformation can be specified and the data will be transformed to this new basis. The Wyckoff positions are specified by the Wyckoff letters, multiplicities, coordinate triplets and site-symmetry groups. Optionally, the symmetry operations of the site-symmetry groups of any point (within the unit cell or specified by its coordinates) can be calculated. Different types of notation are used for the symmetry operations: they are presented as coordinate triplets, in matrix form, using geometric symbols (indicating the type and order of the operations, and the location and orientation of the corresponding geometric elements, and screw or glide components if relevant) and as Seitz symbols. Information is also available for the Euclidean, chirality-preserving and affine normalizers of the space groups.

(ii) Symmetry relations between space groups: The maximal subgroup data given in Volume A1 are extended to subgroups of arbitrary index for all the space groups, and series of isomorphic subgroups are available for indices up to 50 for orthorhombic, tetragonal, trigonal and hexagonal space groups and for indices up to 27 and 125 for cubic space groups. Interactive contracted and complete graphs of chains of maximal subgroups, including basis transformations and origin shifts for each step, can also be generated. In addition, data for supergroups of arbitrary index of all the space groups are provided. In contrast to Volume A 1, where only space-group types of supergroups are indicated, in the symmetry database each supergroup is listed individually and specified by the transformation matrix that relates the conventional bases of the group and the supergroup. The subgroup and supergroup data can be transformed to the basis of the group, left- and right-coset decomposition calculations can be carried out, and Wyckoff-position splittings can be obtained along with the relations between the coordinates of the positions within the group and subgroup.

(iii) 3D Crystallographic point groups: The data for the point groups, presented in an analogous way to the space-group data, include generators, and general and special Wyckoff positions. The data can be transformed to different settings, thus enhancing and extending the data tabulated in Volume A. Clear and instructive visualization of the symmetry elements of the crystallographic point groups and their stereographic projections, including interactive 3D polyhedra representations of idealized crystals, is also provided [3].

The Symmetry Database is available to all subscribers to the online version of International Tables for Crystallography. A Teaching Edition of the Symmetry Database, which can be used to obtain and explore the data for a selected set of space groups is also available online.

[1] International Tables for Crystallography (2016). Volume A, Space-Group Symmetry, 6th ed., edited by M. I. Aroyo. Chichester: Wiley.

[2] International Tables for Crystallography (2010). Volume A1, Symmetry Relations between Space Groups, 2nd ed., edited by H. Wondratschek \& U. Müller. Chichester: John Wiley \& Sons.

[3] Arribas, V., Casas, L., Estop, E. \& Labrador, M. (2014). Comput. Geosci. 62, 53-61.

\section{Keywords: online symmetry database; space-group symmetry; symmetry relations between space groups; crystallographic} point groups

The Symmetry Database has been developed as part of an ongoing project between International Union of Crystallography, eFaber Soluciones Inteligentes SL. (Bilbao) and the Bilbao-Crystallographic-Server team. Most of the additional crystallographic data for the space groups, their subgroups and supergroups, and program algorithms have been provided by the Bilbao Crystallographic Server (www.cryst.ehu.es).

Acta Cryst. (2021), A77, C668 